\title{
A Smart Motor Driving System Using Pu's Count Modulation Multichannel Communication
}

\author{
Wen-Cheng Pu*, Yu-Dian Lin, and Jian-Xing Wu \\ Department of Electrical Engineering, National Chin-Yi University of Technology, No. 57, \\ Sec. 2, Zhongshan Rd, Taiping Dist. Taichung 41170, Taiwan (ROC) \\ (Received May 31, 2019; accepted December 6, 2019)
}

Keywords: Smart motor, PKM, PWM, MPKM, ADALINE, ATMEGA328PU

In this paper, we propose a new-generation motor architecture (smart motor) constructed by first integrating a motor, driver, controller, and communication interface into a control unit, and then using Pu's count modulation (PKM) technology to replace the traditional pulse width modulation (PWM) technology to power a smart motor; the proposed motor architecture and PKM technology have a communication function. The purpose of this study is to virtualize the motor control through the design of the smart motor, while simultaneously driving the heterogeneous motor. However, the proposed PKM technology uses the masterservant communication architecture to transmit the message of only one smart motor at a time. Therefore, as the number of responding smart motors increases, the overall response time is directly increased; this indirectly affects the response sensitivity of the smart motor drive system. Another contribution of this paper is the proposal that the establishment of an ADALINE artificial neural network can facilitate a set of multichannel, parallel communication architectures for computing the signal from a sensor to reduce the overall communication time and increase system response capabilities; this system is called multichannel PKM (MPKM) technology. In the smart motor architecture, the drive system only needs power busbars, which can randomly increase or decrease the number of drive motors, simplify the drive program, control the power signal interference problem, and reduce the number and weight of control lines. At the end of this paper, the signal is analyzed using MATLAB/Simulink ${ }^{\circledR}$, and the microcontroller (MCU) ATMEL328PU is used as the control center. The hardware of the architecture system can be used to verify its feasibility.

\section{Introduction}

The technology of unmanned smartauto plants has been widely studied in recent years as a result of the proposal of the Internet of Things. The power of the unmanned smartauto plant is often supplied by motors, so the multimotor cooperative drive system has become increasingly important. The traditional multimotor drive system, as shown in Fig. 1, is driven by several robotic arms $\mathrm{R} n(n=\mathrm{A}, \mathrm{B}, \mathrm{C}, \ldots)$, simulating the plant environment. However, each robot arm is limited by the number of motors driven by its own controller $x(x=\mathrm{A}, \mathrm{B}, \mathrm{C}, \ldots)$. Therefore,

*Corresponding author: e-mail: puo@ncut.edu.tw https://doi.org/10.18494/SAM.2020.2488 


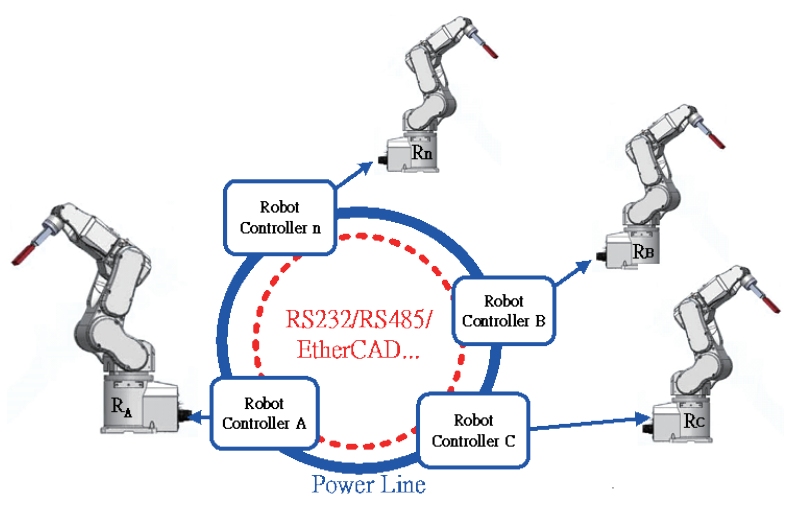

Fig. 1. (Color online) Robot arm drive architecture.

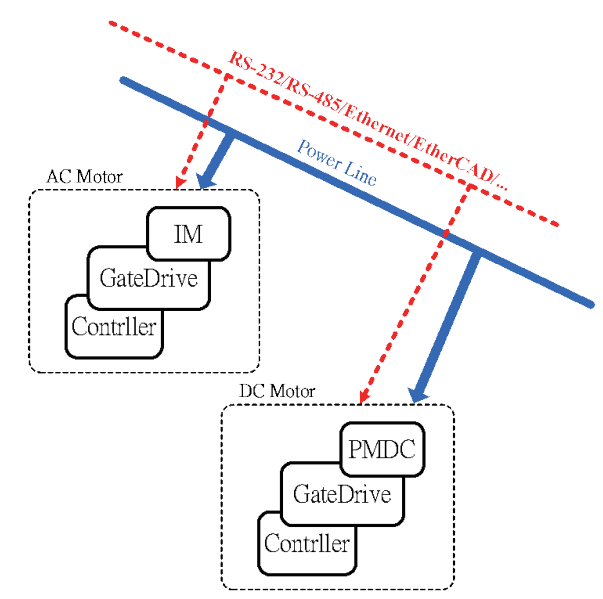

Fig. 2. (Color online) Structure of traditional heterogeneous motor drive system.

as shown in Fig. 1, a collaborative drive must be achieved through interface communications such as Ethernet, EtherCAD, or RS232. ${ }^{(1-7)}$ However, this hides the correctness and stability of the conversion of the control signal flow in different communication interfaces and the response time of the overall drive system. Another problem, as shown in Fig. 2, is that in the classification of heterogeneous motors, although both DC and AC motors have their respective advantages, the corresponding driving methods are different. Thus, we may first need different control panel drivers, ${ }^{(3-7)}$ as described above, and then use the communication interface to integrate and coordinate the driving; however, this makes the process complicated. Considering the motor driving technology and the differences between the AC motor and the DC motor, the overall drive technology is far more complex. The simplification of the coordination process and the technology of the motor virtualization drive are highly important because the multiheterogeneous motor drive technology is necessary for intellectualization.

As shown in Fig. 2, motors are mainly categorized as DC and AC motors. Although each category has its own advantages and disadvantages, the main problem is that the corresponding driving methods are different, making it impossible to drive both at the same time. However, it is found that pulse width modulation (PWM) technology is used as the driving core technology in the two types of motor driving technology.

The traditional PWM technology involves controlling the carrier frequency so that highfrequency noise can be easily filtered by the low-pass-rate filter. ${ }^{(8)}$ However, because it can cause motor vibration, in practice, the limit of the carrier frequency should not be too low. Another point of view is that the fundamental wave of the low carrier frequency is not spaced apart from the harmonic frequency, which makes the bandwidth design of the wave filter very difficult; furthermore, it is not easy to eliminate the harmonic frequency, and the volume of the wave filter is relatively large. Another aspect of this study involves the exploration of techniques for improving efficiency and eliminating specific harmonics. Among the research themes, ${ }^{(8,9)}$ the main techniques involve the exploration of how to control the turn-on and turnoff times to eliminate specific harmonics. Although there is an advantage to eliminating specific harmonics, doing so consumes much computing resources and requires offline 
calculations; therefore, it is not recommended to eliminate too many high-order harmonics. In Refs. 8 and 10, the authors used the controlled the space vector to eliminate the harmonics and increase the overall efficiency. However, the calculation consumes too much computational resources, so that the main disadvantage is that this method cannot eliminate too many specific harmonics simultaneously. ${ }^{(8-10)}$ From Refs. 8 to 11 , the authors also adopted a PWM circuit module design in the hardware used to eliminate the specific harmonic component and increase efficiency through the coordination of conduction time. However, the aforementioned studies mainly investigate techniques for eliminating harmonics and increasing efficiency but do not discuss the heterogeneous motor collaborative driving technology and communication. Furthermore, the number of controllers is limited by the independent characteristics of the controller and various communication interfaces. The multimotor homogeneous cooperative system has both the AC motor and the DC motor, i.e., difference-driven technology, so that the different types of motor were driven by different methods, and the control between the heterogeneous motors is complicated. When the commands are transmitted between different communication interfaces, the accuracy of the command conversion varies with the different specifications adopted, let alone the long response time. Therefore, in this research, we first propose a communication technology, Pu's count modulation (PKM) technology to solve the problems encountered when using the PWM technology without communication capability. Second, PKM is further used to solve the harmonic control problem. Additionally, in this paper, we propose a new drive interface for smart motors to remove the limits on the number of controller axes and simplify the wiring and control methods. The main purpose of this paper is to enable the driving of heterogeneous motors on the same platform.

In addition, the control system that uses an EtherCAD communication interface transmission control command is the most popular communication interface, and its response time is relatively short. The EtherCAD communication interface mainly refers to the Ethernet communication interface specification and OSI network communication architecture. However, it uses the special-case rules applied to the package in the IEEE802 specification adopted by the Ethernet so that the same package can contain an array of motor drive commands without being limited by the traditional Ethernet package, which can only transmit one set of motor control commands at a time. This speeds up the response time of the drive motors. To reduce the response time, the ADALINE artificial neural network was employed to establish a parallel PKM communication system, which can transmit multiple commands from each smart motor simultaneously, thus reducing the overall response time of the motor drive system.

This paper is organized as follows. The techniques used in this study are explained in Sect. 2. In Sect. 3, the test platform is established and the theoretical simulation and implementation proposed are described. In the final section, a conclusion is drawn and the direction of future applications is explained.

\section{Materials and Methods}

To simplify the coordination process and motor drive, in this paper, we adopt the notion of the object-orientation concept and propose the architecture of the smart motor. As shown in 
Figs. 3(a) and 3(b), the motor drive, driving controller, driving function, and communication interface are packaged into a single unit called a smart motor unit. Figure 3(a) shows the smart motor architecture of a packaged DC motor, and Fig. 3(b) shows the smart motor architecture of a three-phase AC induction motor. Therefore, regardless of the smart motor being packaged with a DC or AC motor, the smart motor is just an object outside and only needs to send a command via the communication interface; this is the first contribution of the smart motor design that enables the virtualization of the motor drive.

In addition, as shown in Fig. 2, the power supply and communication module are designed separately in a traditional heterogeneous motor hybrid-driven application, but the heterogeneous motor rectifies the primary power into DC and the busbar of DC provides the power. Then, the proposed PWM technology is used for the drive motor, as shown in Fig. 4(a) or 4(b). For example, the packaged DC motor, as shown in Fig. 3(a), is driven by PWM power, as shown in Fig. 4(b). If $V_{d}, v_{a}, Q_{1}, Q_{2}, Q_{3}$, and $Q_{4}$ represent the DC busbar voltage, the DC motor input voltage, and the power switches, respectively, the armature current $i_{a}$ can be calculated using Eq. (1).

$$
i_{a}(t)=\Delta i_{a}+I_{a}=\frac{v_{a}}{2 R_{a}}\left[\frac{1+e^{\frac{T}{\tau_{a}}}-e^{\frac{\delta T}{\tau_{a}}}-e^{\frac{(1-\delta) T}{\tau_{a}}}}{e^{\frac{T}{\tau_{a}}}-1}\right]+\frac{v_{a}}{2 R_{a}}\left[\frac{e^{\frac{T}{\tau_{a}}}+e^{\frac{\delta T}{\tau_{a}}}-e^{\frac{(1-\delta) T}{\tau_{a}}}-1}{e^{\frac{T}{\tau_{a}}}-1}\right]-\frac{E}{R_{a}}
$$

$T, E, \tau_{a}$, and $R_{a}$ represent the period, the induction voltage, the response time constant of the static coil, and the static coil resistance, respectively. The result of the calculation can be used to calculate the vibration torque and steady-state electromagnetic torque $\left(T_{e}\right)$ of the operation. The vibration torque, which is also called the disturbance torque, can cause vibration and noise and is calculated according to Eq. (1), where $\delta=t_{o n} / T$, which is called the duty ratio. $k$ and $\varphi$ represent a constant and static flux, respectively.

$$
\Delta T_{e}=k \varphi \Delta i_{a}=\frac{k \varphi v_{a}}{2 R_{a}}\left(e^{\frac{1}{2 f_{s} \tau_{a}}}-1\right)
$$

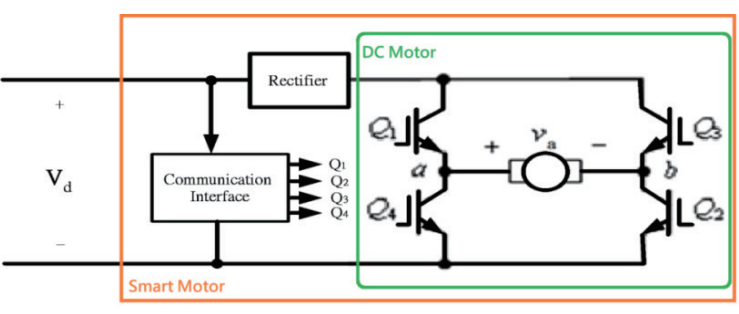

(a)

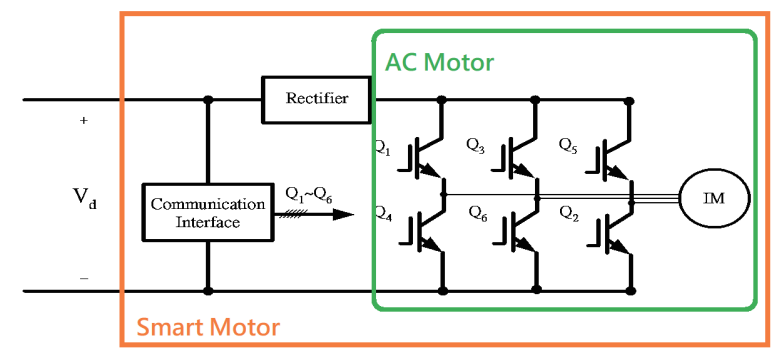

(b)

Fig. 3. (Color online) Architecture of smart motor. (a) Single-unit architecture of smart motor packaged with DC motor. (b) Single-unit architecture of smart motor packaged with three-phase induction motor. 


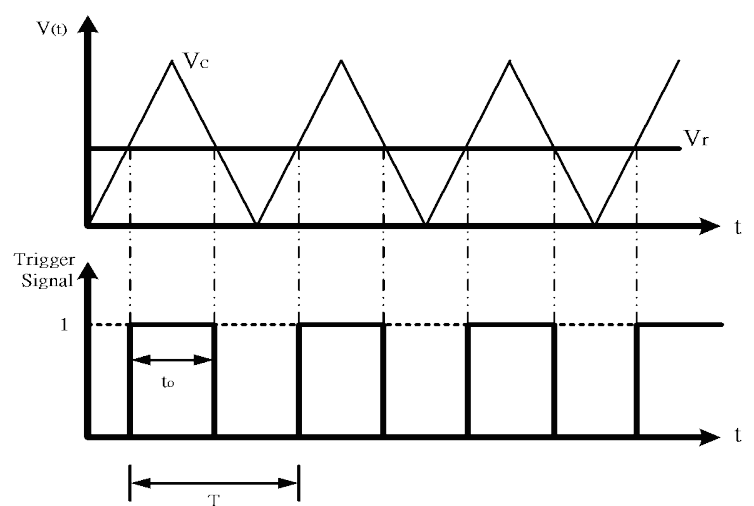

(a)

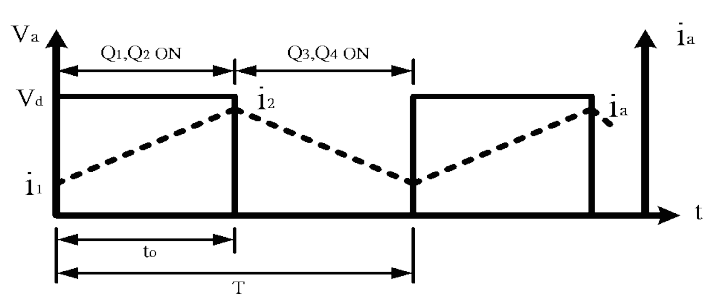

(b)

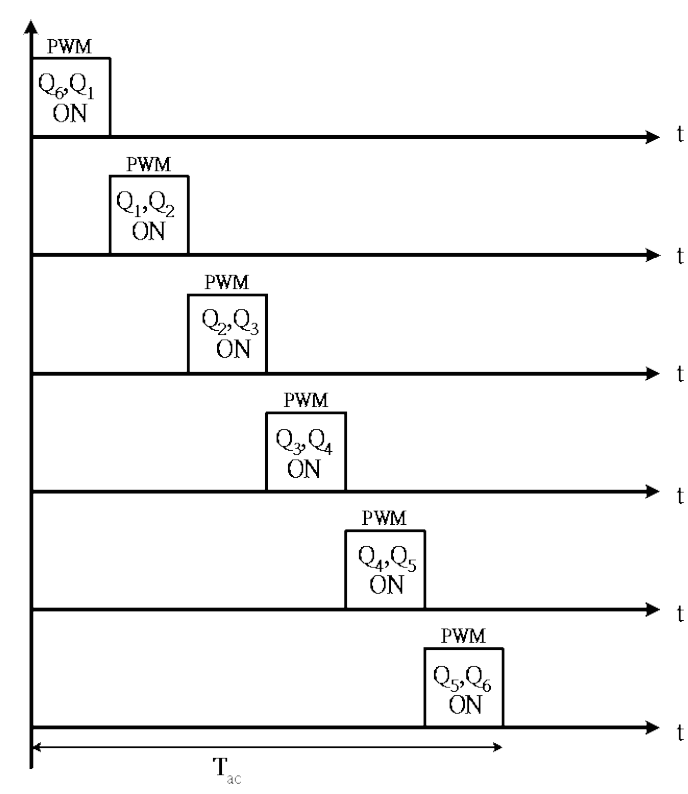

(c)

Fig. 4. PWM cycle operation. (a) PWM modulation method and waveform. (b) Current waveform during PWM period. (c) AC-motor drive strategy.

Equation (2) demonstrates that the vibration is mainly determined by the frequency of the carrier, $f_{s}(=1 / T)$. In practice, the vibration can be reduced by increasing the carrier frequency. However, when a higher frequency is selected, although the vibration decreases, the loss during power switching increases, ${ }^{(11)}$ and the system efficiency is reduced. Selecting the right $f_{s}$ is another research topic. In addition, the equivalent armature voltage $v_{a}$ and the electromagnetic torque $T_{e}$ can be calculated according to Eq. (3) after stabilization, where $\omega_{s}=6.28 \mathrm{fs}$ and $t_{o}$ is the conduction time in every period $T$.

$$
\begin{gathered}
v_{a}=\lim _{n \rightarrow 0} \sum_{n=-\infty}^{n=\infty} \delta V_{d} \frac{2 \sin \left(\frac{n \omega_{s} t_{o}}{2}\right)}{n \omega_{s} t_{o}} e^{-j n \omega_{s} t}=\delta V_{d} \\
T_{e}=k \varphi I_{a}=k \varphi\left(\frac{\delta v_{a}-E}{R_{a}}\right)
\end{gathered}
$$

As shown in Fig. 3(b), the PWM power wave can also drive a three-phase AC motor using a six-square wave strategy. Although heterogeneous motors are driven by PWM technologies, different switching frequencies may interfere with communication, affect the communication quality, and cause harmonics resulting in a power system malfunction. ${ }^{(8)}$ As such, the final conclusion is that PWM power waves are not capable of communication. Another purpose 
of the smart motor design is to ensure that in addition to providing power, the traditional DC busbar can be modified to provide the capability of two-way communication. However, as shown in Fig. 2, the use of additional communication interfaces can make the buster susceptible to power interference by high-power motors; furthermore, the buster requires additional proprietary circuitry. Although the traditional power line carrier technology, which requires additional small communication signals to the power signal, is widely used in Europe and the United States, it requires additional hardware, and the quality of communication can easily be affected by the unstable electrical load structure and the motor core saturation phenomenon. ${ }^{(12-14)}$ In this study, to solve these problems, as shown in Fig. 5, we will replace the traditional DC busbar with a new PKM busbar, which adjusts the DC busbar voltage with PK coding technology, and establish a driving platform for the smart motor. Not only can the PKM power wave provide the smart motor with power, the built-in PK code can also transmit commands to each smart motor. Therefore, to drive the smart motor packaged with the DC motor or to drive the smart motor packaged with an AC induction motor, only a front controller comprising H-bridge circuits should be added. The structure of the front controller converts the voltage output of the traditional DC busbar voltage into a PKM power wave $V_{d}$ with the non-return-tozero (NRZ) feature and reoutput. This structure is called the PKM busbar. The smart motor on the PKM busbar is divided into two parts: a rectifier and a communication interface through the PKM power wave. The communication interface receives the command transmitted by the PKM power wave and drives the motor by the selected method packaged in the smart motor when the command is received, such as a six-square wave strategy or a vector control strategy, and the rectifier is responsible for restoring the PKM power into DC power, providing motor drive power, and driving the packaged motor according to the command received by the communication interface. ${ }^{(15)}$ The relevant technologies are explained as follows.

\subsection{PK coding strategy}

The PK coding is a digital coding technology introduced in recent years. ${ }^{(16)}$ Its architecture is shown in Fig. 6, and it is mainly divided into two areas: data zone and error zone. The

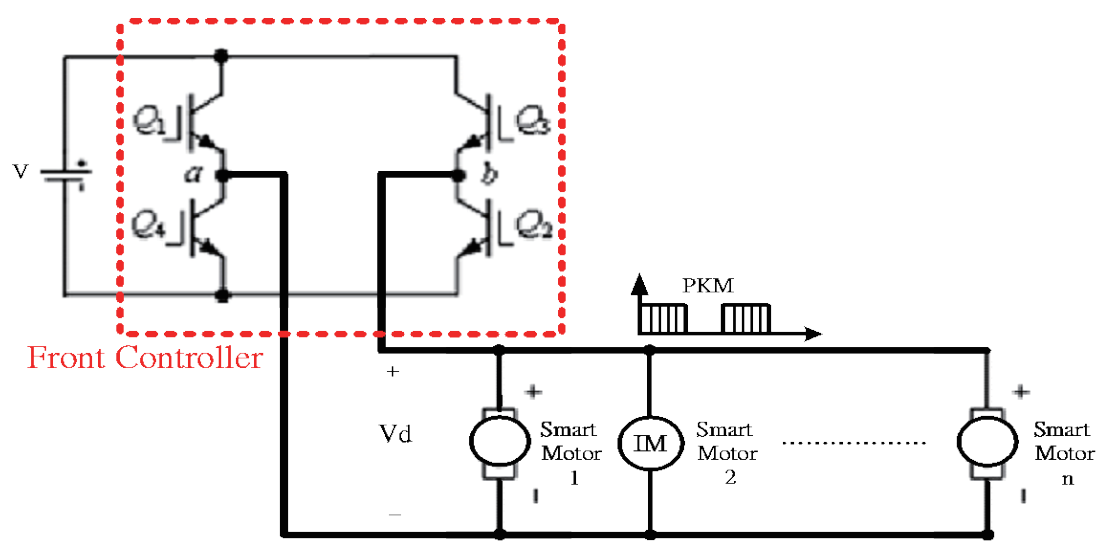

Fig. 5. (Color online) Architecture of smart motor drive system. 


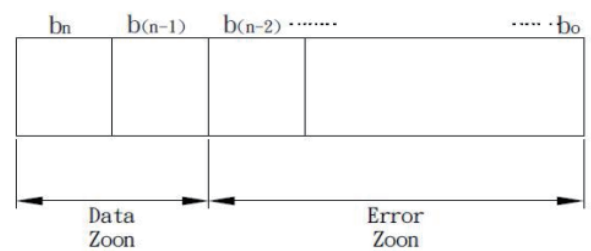

(a)

\begin{tabular}{ccc}
\hline$b_{n}$ & $b_{(n-1)}$ & Description \\
\hline 0 & 0 & Undefined \\
0 & 1 & Bit data "0" \\
1 & 0 & Bit data "1" \\
1 & 1 & Stop bit \\
\hline
\end{tabular}

(b)

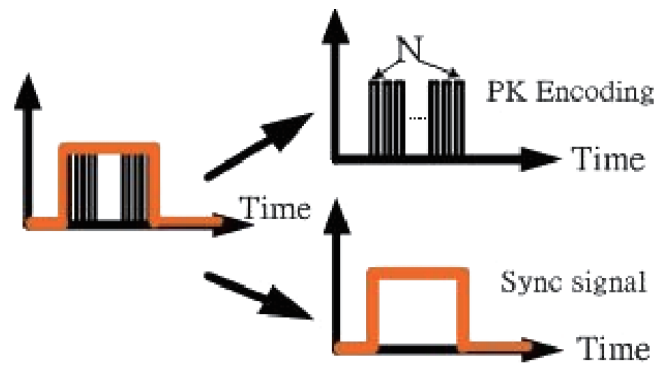

(c)

Fig. 6. (Color online) PK coding: (a) architecture of coding, (b) conversion table of data zone, and (c) wave form of coding.

data zone uses bits as its unit, converts the data of "1" and "0", as shown in Fig. 6(b), into binary data $\kappa$ with two bits, and brings in the value of $\kappa=$ " $b_{n} b_{(n-1)}$." The error zone, on the other hand, is responsible for preventing errors in the communication process, as shown in Fig. 6. In the transmitting environment, the relative error level coefficient $\varepsilon$ of the binary is adopted as $\varepsilon=2^{\gamma}, \gamma=n-2$ (Table 1) and is brought into the error zone shown in Fig. 6(a). The $\varepsilon$ value was selected on the basis of the transmitting environment, and it can be determined easily from communication strategies. Although larger $\varepsilon$ values make transmission results more accurate, we selected $\varepsilon=8, \gamma=3$, to reduce the communication time to the extent that correctness is acceptable. Finally, by filling the package shown in Fig. 6(a), we can calculate the corresponding decimal value in $N m$ with 8421 coding, as in Eq. (5).

$$
N m=\kappa \times \frac{3}{2} \varepsilon
$$

If $f_{s}$ is defined as the speed of the communication pulse wave generator on the transmission side, we place no limitations on the format of the pulse wave [such as return-to-zero (RZ), NRZ, and sine wave shape] and calculate the corresponding values in $\mathrm{Nm}$ with PK coding to control $f_{s}$. After calculating the number of pulse waves, the receiving end only needs to unconditionally select the integer obtained by dividing the number of pulse waves by $\varepsilon$, which is called PK decoding, to infer the transmitted data. The transmission speed, represented by $\mu$, can be calculated from Eq. (6). In other words, the receiver only needs to calculate the number of pulse waves, and it will be automatically compatible with different communication bandwidths during the continuity process.

$$
\mu=\frac{f_{s}}{N m} \text { bps }
$$


Table 1

Relationship between error level coefficient and transmitting environment.

\begin{tabular}{lc}
\hline Transmitting environment & Relative error level coefficient \\
\hline Unacceptable & Above $2^{\gamma}, \gamma>4$ \\
Inferior & $2^{4}$ \\
Normal & $2^{3}$ \\
Good & $2^{2}$ \\
Excellent & $2^{1}$ \\
\hline
\end{tabular}

After PK encoding, the output is a series of waves; in addition to transmitting data, it also hides the synchronized signal since the low-frequency portion is the synchronized signal. As shown in Fig. 6(c), we can separate the synchronized signal very easily, which improves the traditional synchronized communication technology using synchronized frames or additional synchronized clock signals. However, the number of pulse waves changes owing to interferences during the transmission, but the receiving side only needs to calculate the number of pulse waves, and the numbers is in the range of $\left[\kappa \times 2^{\gamma}+\left(2^{\gamma}-1\right)\right]-\left(\kappa \times 2^{\gamma}+0\right)$. After PK decoding, the transmitted data can be correctly restored. $P e$ is defined as the probability of error, which is calculated using Eq. (7), but it shows that the receiving end can keep correct data in the data zone even if error occurs, so the PK coding system is fault-tolerant.

$$
P_{e}=\frac{2^{\gamma+1}-1}{2^{\mu+\gamma}}
$$

Finally, by transmitting a continuous sequence of pulses $\kappa=" b_{n} b_{(n-1)} "=$ "1 1 " on the transmitting side, the transmission is ended. Hence, the receiving end can automatically determine the length of the data during communication.

The PK digital coding technology, with a bit-based coding unit, has a simple coding structure and a debugging function. The encoded signal conceals the synchronized signal, allowing the communication receiver to automatically recognize the length of the transmitted data. Moreover, it can be automatically recognized and be compatible with different communication speeds. Therefore, it can be compatible with various protocols and can replace the physical layer coding technology. ${ }^{(15,16)}$

\subsection{PKM technology strategy}

The smart motor drive platform is designed with a PKM busbar voltage $V_{d}$. In addition to providing power to the smart motor that is superimposed on the platform, the PKM busbar must also be used as the communication medium, as shown in Fig. 7. After the front controller converts the data to be transmitted into PKM power waves, the signal flows as shown below. The front controllers that are responsible for the busbar DC voltage of the traditional rectified output, the $\mathrm{Nm}$ value encoded according to the $\mathrm{PK}$, and the selected pulse wave format (such as RZ and NRZ) will modulate the DC voltage output, known as a PKM power wave. The spectrum distribution of the power $v_{p}$ from the PKM busbar to the smart motor, most importantly, may cause interference problems along the line. 


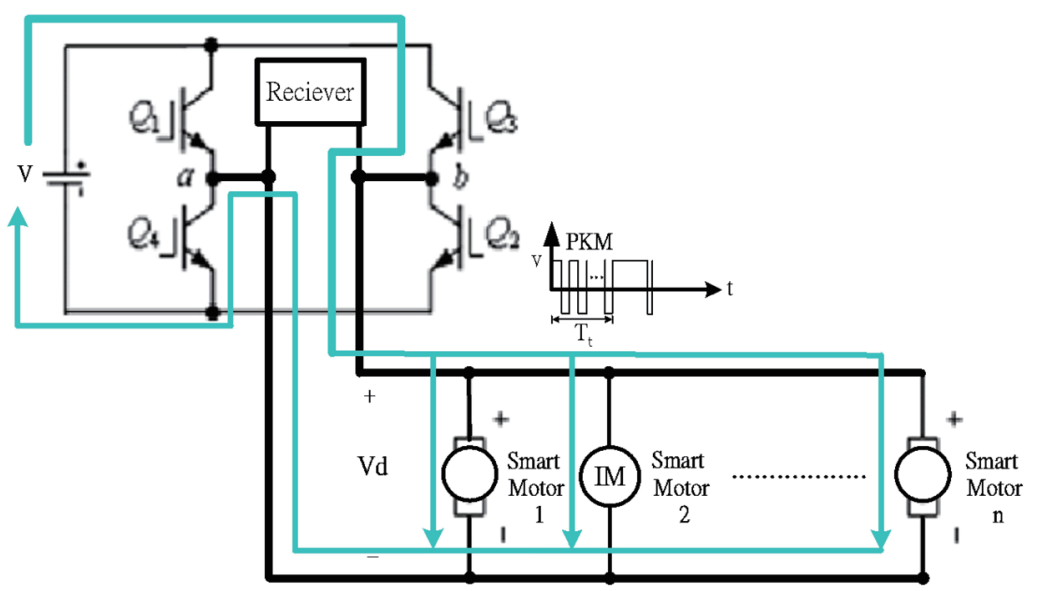

Fig. 7. (Color online) Communication signal transmission path of control command transmitted from front controller to smart motor.

$$
\begin{gathered}
v_{p}(t)=\sum_{n=-\infty}^{n=\infty} c_{n} e^{j 2 \pi n f_{s} t}=\sum_{n=-\infty}^{n=\infty}\left[a_{f s} \cos \left(2 \pi n f_{s} t\right)+b_{f s} \sin \left(2 \pi n f_{s} t\right)\right], n \in N a_{f s}, b_{f s}, c_{f s} \in R \\
c_{f s}=f_{s} \int_{t_{0}}^{t_{0}+\frac{1}{f_{s}}} v_{p}(t) e^{j 2 \pi n f_{s} t} d t \\
a_{f s}=f_{s} \int_{t_{0}}^{t_{0}+\frac{1}{f_{s}}} v_{p}(t) \cos \left(2 \pi n f_{s} t\right) d t \\
b_{f s}=f_{s} \int_{t_{0}}^{t_{0}+\frac{1}{f_{s}}} v_{p}(t) \sin \left(2 \pi n f_{s} t\right) d t
\end{gathered}
$$

The power $S$ of the PKM power wave $v_{p}$ is

$$
S=f_{s} \int_{t_{0}}^{t_{0}+\frac{1}{f_{s}}} v_{p}^{2}(t) d t=a_{0}^{2}+\sum_{n=1}^{n=\infty} \frac{a_{n}^{2}+b_{n}^{2}}{2}=2 c_{n}^{2},
$$

and the efficiency can be calculated using Eq. (10).

$$
\eta=\frac{S_{f_{s}}}{\sum_{n=0}^{n=\infty} S_{n f_{s}}}
$$

To adopt the communication capabilities, PKM uses periodic NRZ to concentrate harmonics and control interference from other frequencies in the environment. As such, according to Eq. (8), Fourier analysis of the Greek wave component is carried out. Figure 8 shows the wave that the DC busbar rated voltage $V$ is modulated with communication pulse wave $f_{s}$, and the pulse 


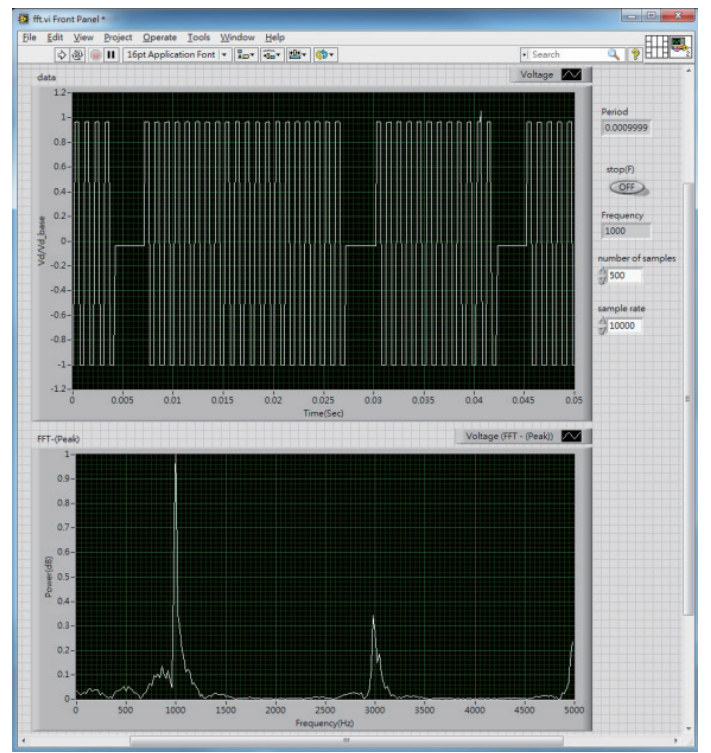

(a)

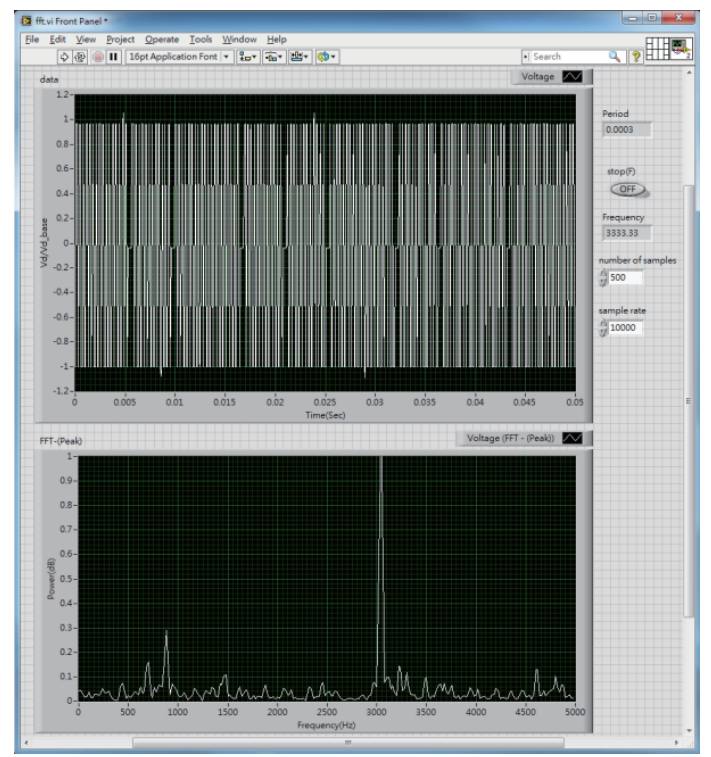

(b)

Fig. 8. (Color online) PKM power waves using different pulse frequencies $f_{s}$ to transmit data "K" ("0100 1011"), and its frequency spectrum distribution. (a) $f_{s}=1 \mathrm{kHz}$. (b) $f_{s}=3 \mathrm{kHz}$.

frequencies $f_{s}=1 \mathrm{kHz}$ and $f_{s}=3 \mathrm{kHz}$ are selected to transmit the spectrum distribution of the PKM power wave $v_{p}$ with the ASCII code "K". The verification Eq. (8) shows the correctness of the spectrum distribution, and we found that the frequency distribution is more concentrated. Moreover, the distribution indicates that as the PKM power wave transmits data, we set $n=0$, and the DC value can be obtained using Eq. (11),

$$
v_{p}^{d c}=\sum_{n=0}\left[\frac{4 v}{n \pi} \sin \left(2 \pi n f_{s} t\right)\right]=\frac{1}{\pi} \int_{t=-\pi}^{t=\pi} v_{p}(t) d t=c_{0} e^{j 0.2 \pi f_{s} t}=0
$$

which is approximately zero. Thus, the DC loss is reduced, which indirectly indicates an increase in efficiency. Figure 8 shows its authenticity and indirectly indicates that the main harmonic distribution of the PKM power wave form may change according to the communication pulse wave $f_{s}$, but changing the communication pulse wave $f_{s}$ will not affect its communication capability, which indicates that while the PKM power wave is maintaining communication capability, as long as the communication pulse $f_{s}$ on the transmission side is changed, we can control the electromagnetic wave interference bandwidth discharged into space. Changing the distribution of the spectrum can effectively control the external interference source of electromagnetic waves to avoid the frequency bands that easily interfere with the surrounding environment. In the spectrum shown in Fig. 8, the other frequencies are caused by the time interval of each PKM power wave. Therefore, in general, the implementation may still change the frequency of the communication pulse wave to avoid the frequency of environmental interference, or to adjust the power switch and $\Delta T_{e}$. 
However, although the PKM technology uses the communication pulse wave $f_{s}$ to modulate communication data, the changing communication pulse wave $f_{s}$ does not affect the reliability of communication. ${ }^{(15,16)}$ This is the most satisfying characteristic of PK communication and what the existing communication technology has yet to achieve. In addition, the reason for selecting the PKM power wave of the NRZ format is that $v_{p}$ can be rectified into the DC power after passing through the rectifier, which provides power to the smart motor. To achieve the purpose of two-phase communication, the simplest method is to employ a communication frequency as a communication channel (channel) to transmit commands; it is more suitable to use the master-servant half-duplex communication mode. Moreover, within each communication, the package can only be responded to by a single smart motor. As the response time of the smart motor increases, the overall response time increases, which is the only limitation in a channel. In addition, because the PKM power wave is a digital signal, the PK encoding and decoding processes do not need to be calculated using a floating point, which is suitable to the microcontroller (MCU) without a float computing unit. The encoding does not require high computational capability. Therefore, a simple low-cost single-chip processor accomplishes the task, which can improve the encoding and decoding efficiencies, reducing the costs of computing and hardware. This is another advantage of PK encoding technology.

\subsection{Establishing a multichannel communication strategy with ADALINE artificial neural network ${ }^{(16-20)}$}

According to the EtherCAD communication interface, to reduce the overall drive response time, the communication package format of the Ethernet interface should be modified so that the one-time transmission package can control multiple motors. The communication mode of the smart motor adopts a master-servant structure, but when data are sent back, they can only be sent in batches in series. As the number of smart motors in the system increases, the overall reaction time of the system will randomly increase. To solve this problem, in this study, we use an ADALINE artificial neural network to establish a multichannel communication system. In a single hardware circuit, parallel communication technology is adopted. Fixing communication time alone will allow a random number of smart motors to send back information, thus greatly reducing the overall response time of the drive platform of the smart motor. The ADALINE artificial neural network architecture is shown in Fig. 9. According to the Fourier series concept, a periodic function $y$, as shown in Eq. (12), can be composed of different orders of harmonics. If the returned data are treated as a weight, then each smart motor can use different harmonics as the only communication channel; that is, the $\boldsymbol{W}$ matrix represents the parallel communication architecture of $n$ communication channels.

$$
y(t)=\boldsymbol{W} \boldsymbol{X}^{\mathrm{T}}
$$

In the above equation, $y(t)$ represents a periodic timely signal, which is a $1 \times 1$ matrix; $\boldsymbol{W}$ is a weight matrix; $\boldsymbol{X}$ is an input function matrix, where $\omega$ is the angular speed. 


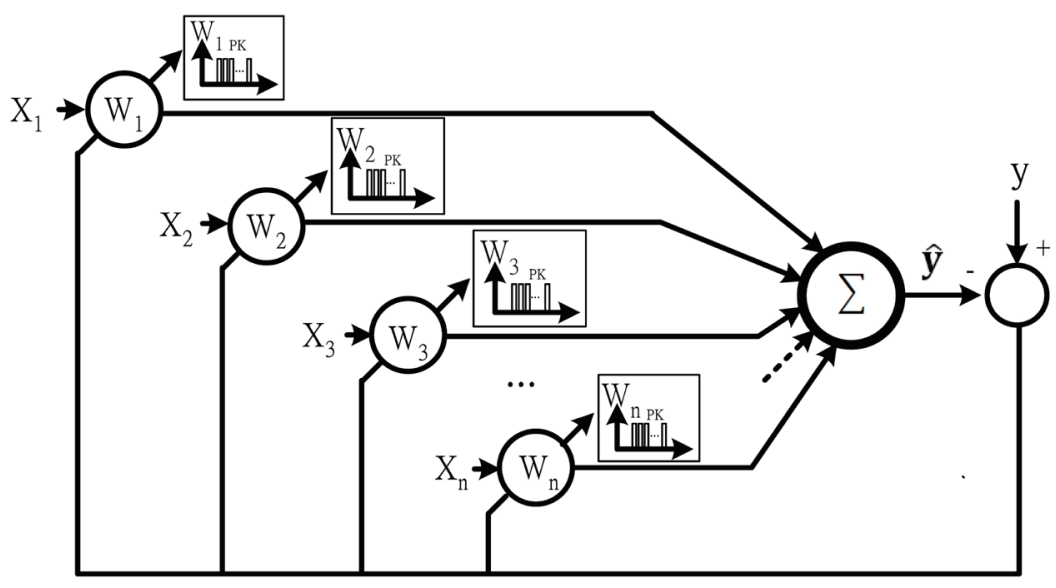

Fig. 9. Architecture of ADALINE artificial neural network.

$$
\begin{aligned}
\boldsymbol{X} & =[1, \sin \omega t, \cos \omega t, \sin 2 \omega t, \cos 2 \omega t, \ldots, \sin (n \omega t), \cos (n \omega t)] \\
& =\left[x_{0}, x_{1}, x_{2}, \ldots, x_{n}\right] \\
\boldsymbol{W} & =\left[w_{0}, w_{1}, w_{2}, \ldots, w_{n}\right] .
\end{aligned}
$$

Here, $w_{i}(i=0,1,2,3, \ldots)$ is the weight component relative to $x_{j}(j=0,1,2,3, \ldots)$. Since the $\boldsymbol{X}$ matrix is a harmonic matrix, each unit component represents an input function, and since the frequency response has a linear independent characteristic, $\int_{0}^{2 \pi} \boldsymbol{X} \boldsymbol{X}^{\mathrm{T}} d \omega t$ is a diagonal matrix. However, according to the theory, estimating the value of the coefficient matrix $\boldsymbol{W}$, as shown in Eq. (13), requires that you must first sample $n$ points to form a signal extraction window (here after called Window), and calculate $\boldsymbol{W}$ according to Eq. (15).

$$
\begin{gathered}
{\left[\begin{array}{lll}
y^{1} & \ldots & y^{n}
\end{array}\right]=\left[\begin{array}{lll}
w_{0} & \ldots & w_{n}
\end{array}\right]\left[\begin{array}{ccc}
x_{0}^{1} & \cdots & x_{n}^{1} \\
\vdots & \ddots & \vdots \\
x_{0}^{n} & \cdots & x_{n}^{n}
\end{array}\right]} \\
\boldsymbol{Y}=\boldsymbol{W}\left(\boldsymbol{X}^{\mathrm{T}}\right)^{n} \\
\boldsymbol{W}=\boldsymbol{Y}\left[\left(\boldsymbol{X}^{\mathrm{T}}\right)^{n}\right]^{-1}
\end{gathered}
$$

By the signal capture (Window) method, the weight matrix $\boldsymbol{W}$ can be calculated, but the biggest disadvantage is the waste of too much computing resources. To solve this problem, the ADALINE artificial neural network shown in Fig. 9 is used in this study to replace the signal capture window. The ADALINE architecture is simple and suitable for linear independent estimation systems. Therefore, if $\Delta \boldsymbol{W}$ represents the overlay update item, then $E$ is a square value of error between the estimation variable $\hat{y}$ and the target variable $y$ as objective functions 
that can be set. Equations (16) to (21) show the use of the Widrow-Hoff learning principle to update the weight value $\boldsymbol{W}$.

$$
\begin{gathered}
\hat{y}=\hat{\boldsymbol{W}} \boldsymbol{X}^{\mathrm{T}} \\
y=\hat{y}+e=\hat{\boldsymbol{W}} \boldsymbol{X}^{\mathrm{T}}+e \\
E(\boldsymbol{W})=e^{2}=(y-\hat{y})^{2} \\
\nabla_{E}^{T}=\left[\frac{\partial E(w)}{\partial \boldsymbol{W}}\right]=-2 e \boldsymbol{X} \\
\boldsymbol{W}(n+1)=\boldsymbol{W}(n)+\Delta \boldsymbol{W}(n) \\
\Delta \boldsymbol{W}(n)=\eta d \nabla \boldsymbol{E}=\eta e(n) \boldsymbol{X}(n)
\end{gathered}
$$

In the equation, “ ^" represents the estimated value, and " $\eta$ " is the learning ratio, which determines the speed and accuracy of convergence. In the formula, $d$ and $\nabla \boldsymbol{E}$ are the denoted vector values and gradient matrix, respectively. They respectively represent the vector in the weight vector $\boldsymbol{W}$ space and the normal vector in the objective function space of the linear transformation. Therefore, each smart motor will select a channel frequency and convert the timely information into a weight value; let each element of $\boldsymbol{W}$ present a channel to transmit command. The front controller uses the ADALINE artificial neural network for overlay to obtain the weight values of each channel. After PK decoding, the response data of multiple smart motors are received during one period. This reduces the overall system response time of the multimotor drive system while increasing the overall reaction speed.

\subsection{Communication strategy from smart motor with PKM power wave}

According to the induction principle, the currents with different frequencies respond independently, as shown in Fig. 10. In the driving platform of the smart motor, regardless of whether we use the smart motor architecture shown in Figs. 3(a) and 3(b), the current calculated by Eq. (1) flows through the rectifier from the power side. The current adopted by different smart motors will flow through the front controller; therefore, it is only necessary to take the waveforms of each frequency, and the basic driving features of each smart motor can be analyzed using Eqs. (1) to (4). The traditional PWM technology does not have the communication capability, but it is widely used in the drive control of heterogeneous motors. When the PWM power wave, as shown in Fig. 4, is included in Eq. (3), we can obtain the average voltage value $\delta V=t_{o n} / T \times V$. In other words, at a fixed PWM carrier frequency, the PWM technology will allow the control of the output equivalent average voltage $\delta V$ by adjusting the operation time $t_{o n}$. The PKM technology has a communication function, but for this 


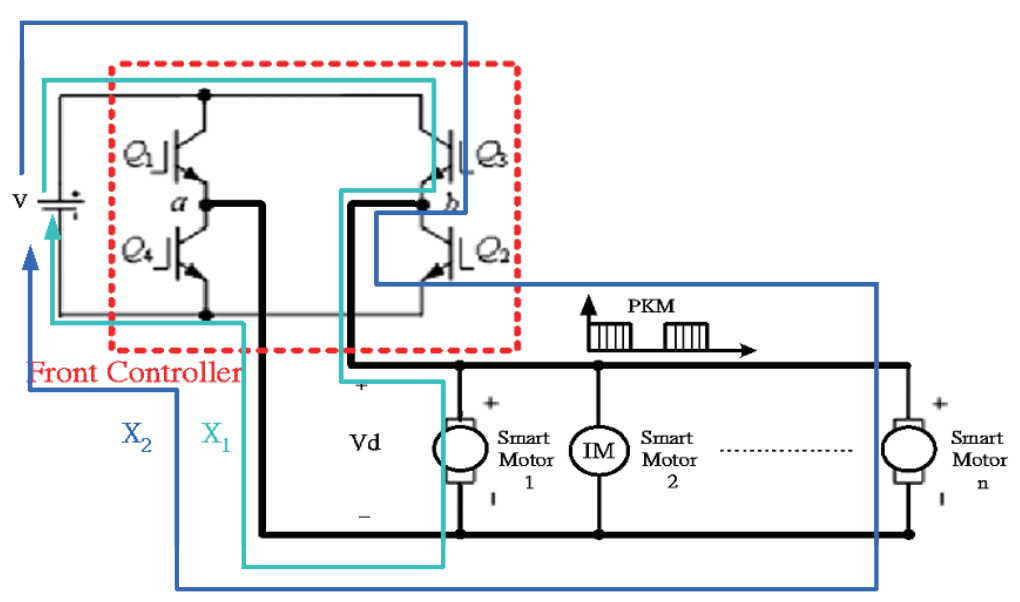

Fig. 10. (Color online) Current flow path in driving platforms for smart motors.

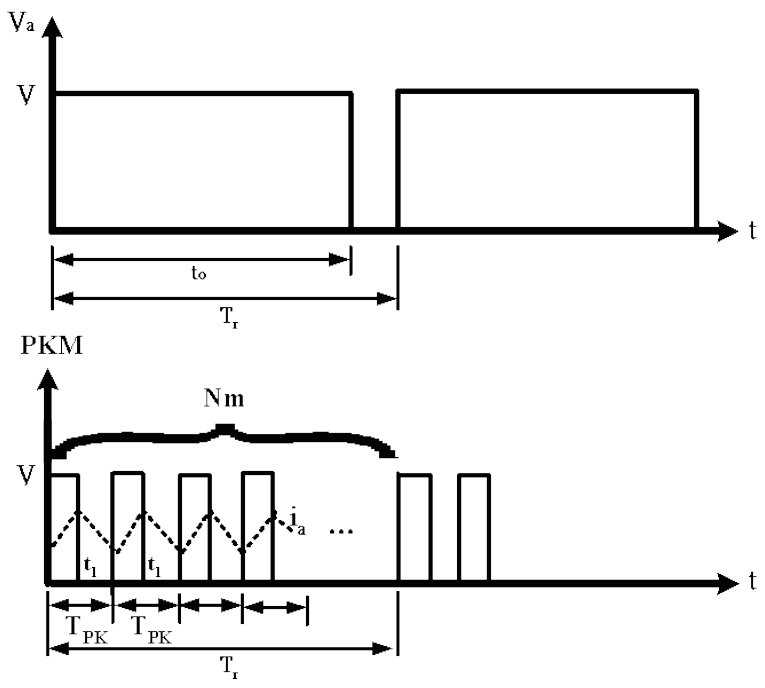

Fig. 11. Interior PKM technology driving strategy for smart motor in comparison with PWM technology.

thchnology to replace the PWM technology, it firstly needs to satisfy the control function of an equivalent average voltage. Fortunately, during communication, the PKM technology only focuses on the correct number of pulse waves, and does not focus on the duty ratio $\delta$ of pulse waves. As shown in Fig. 11, the smart motor follows the received command and converts the received data of the front controller into a PKM power wave to drive the packaged motor.

A traditional PWM power wave is replaced by a series of continuous PKM power waves with the frequency of $f_{S}\left(=1 / T_{P K}\right)$. The responsiveness ratio of each pulse is $\delta_{P K}=t_{1} / T_{P K}$; therefore, the motor output equivalent voltage can be controlled as $\delta_{P K} V$, and the whole output equivalent voltage $v_{a}^{T}$ is

$$
v_{a}^{T}=\frac{N m \times t_{1} \times V}{T}=N m \times \frac{T_{P K} \times t_{1} \times V}{T \times T_{P K}}=N m \times \frac{T_{P K}}{T} \times \delta_{P K} V .
$$


As shown in Fig. 11, taking Eq. (1) as a reference, the input current $i_{a}$ contains the $\Delta i_{a}$ component, which varies with the pulse period, and corresponds to the number of pulses after PK encoding. Therefore, the front controller can capture the pulse of the frequency and conduct PK decoding. Then, as shown in Fig. 12, the ADALINE artificial neural network inside the receiver is used to obtain the sent-back current signal from each smart motor by PK coding, and the sent-back current signal can be obtained using the current sensor.

The strategy of the entire communication cycle is shown in Fig. 13. It is a PKM communication cycle with $T$ architecture that uses code-division multiple access (CDMA) specifications, including the time $T_{t}$ of program transmission and the time $T_{r}$ of program reception. In the time period of $T_{t}$, the front controller outputs commands to the smart motor; in the time period of $T_{r}$, by using the ADALINE artificial neural network, the front controller receives information sent back by the smart motor. There is no need to set a detailed launch time of each program because each transmission data has a cutoff at the end, which can direct the receiving side of the ending transmission to stop and increase the compatibility of different data lengths and specified applications.

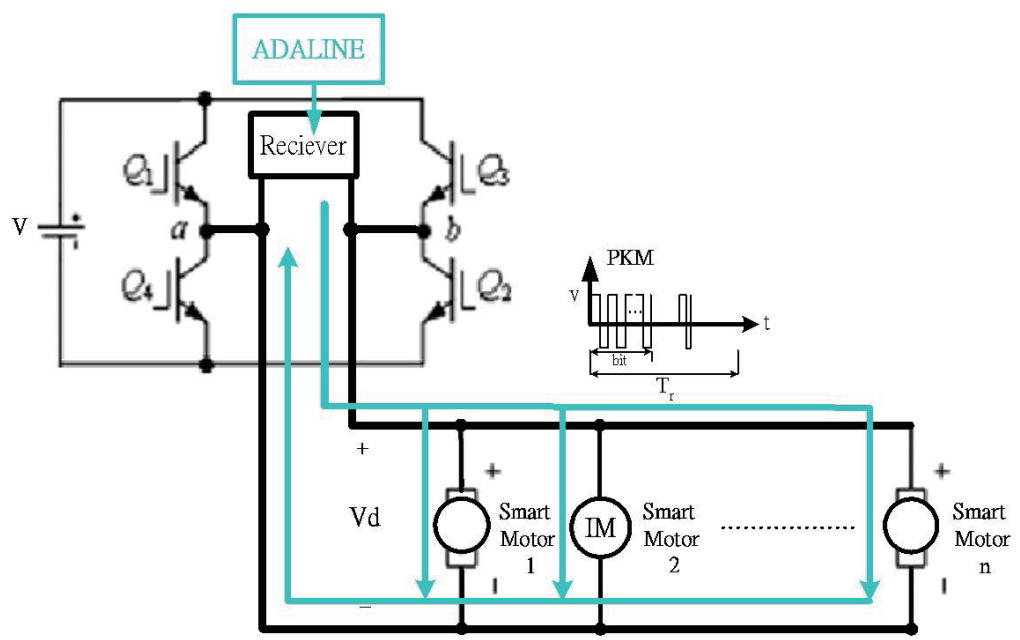

Fig. 12. (Color online) Communication signal transmission path of smart motor using ADALINE artificial neural network to send back information to front controllers.

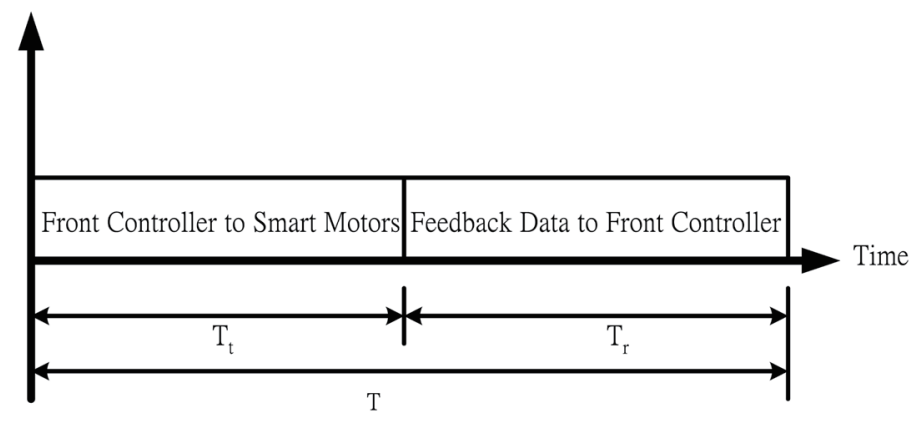

Fig. 13. PKM communication period $T$. 


\subsection{Weight value matrix iteration strategy}

In the ADALINE artificial neural network, Eq. (20) is the correction iteration equation; the normal iteration vector was selected using $\nabla \boldsymbol{E}$ and $\boldsymbol{W}$ on the basis of $d$, and the learning rate $\eta$ will determine the final result and speed of the iteration convergence. The steepest descent method focuses on the direction of the iteration; it chooses to update the weight value matrix along the opposite direction of the normal vector, $(d=-1)$, and lets the object equation satisfy $E(n+1)<E(n)$ after the iteration, as shown in Eq. (12). It applies to the objective equation $E$ when it is a two-dimensional quadratic equation. Because the direction of $-\nabla \boldsymbol{E}$ is always towards where the error $e$ is smallest, the speed of convergence is determined by the learning rate $\eta$. The iteration values of weight must satisfy Eq. (15).

$$
\nabla_{E(n+1)}^{T} \cdot \nabla_{E(n)}=0
$$

In other words, only when $e(n+1)-e(n)<0$ will the iteration process converge, which is

$$
e(n+1)-e(n)=\left[y(n+1)-\boldsymbol{W}(n+1) \boldsymbol{X}^{\mathrm{T}}(n+1)\right]-\left[y(n)-\boldsymbol{W}(n) \boldsymbol{X}^{\mathrm{T}}(n)\right]<0 .
$$

Because the output signal $y$ is a continuous analog signal, when the sampling frequency is high and the sampling duration is sufficiently small, we can assume that

$$
\begin{aligned}
\boldsymbol{X}^{\mathrm{T}}(n+1) & =\boldsymbol{X}^{\mathrm{T}}(n), \\
\boldsymbol{W}(n+1) & =\boldsymbol{W}(n)+\Delta \boldsymbol{W}(n) .
\end{aligned}
$$

When the updated weight matrix is incorporated into the above formula, the condition of convergence is

$$
\begin{aligned}
& y(n+1)-y(n)<[\boldsymbol{W}(n+1)-\boldsymbol{W}(n)] \boldsymbol{X}^{\mathrm{T}}(n), \\
& y(n+1)-y(n)<[\boldsymbol{W}(n)+\Delta \boldsymbol{W}(n)-\boldsymbol{W}(n)] \boldsymbol{X}^{\mathrm{T}}(n), \\
& y(n+1)-y(n)<\Delta \boldsymbol{W}(n) \boldsymbol{X}^{\mathrm{T}}(n), \\
& y(n+1)-y(n)<\eta e(n) \boldsymbol{X}(n) \boldsymbol{X}^{\mathrm{T}}(n) .
\end{aligned}
$$

The convergence range of the learning rate $\eta$ is

$$
\begin{aligned}
& \eta<\frac{y(n+1)-y(n)}{e(n) \boldsymbol{X}(n) \boldsymbol{X}^{\mathrm{T}}(n)}, \\
& 0<\eta<\frac{y(n+1)-y(n)}{e(n)\left(\left[\frac{\|X\|}{2}\right\rceil+1\right)} .
\end{aligned}
$$


The superscript symbol "*” represents the target value, and the error between $y$ and $\hat{y}$ is zero. In addition, the iteration result of the ADALINE is shown in Fig. 14. Owing to the omitted highorder term and noise, the tracked analog signal can cause a stable error $e^{*}$, as inferred from Eq. (28).

$$
e^{*}=y-\hat{y}=y-\hat{\boldsymbol{W}}^{*} \boldsymbol{X}^{\mathrm{T}} \neq 0
$$

Therefore, intuition is very important for the learning rate, and a fixed error value $\alpha$ must be chosen to make $E(\boldsymbol{W})$ approach the $\boldsymbol{W}$ axis. In addition, by observing the following formula, we can infer that

$$
\frac{\partial E\left(\boldsymbol{W}^{*}\right)}{\partial \boldsymbol{W}}=0
$$

Therefore,

$$
\frac{\partial E(\boldsymbol{W})}{\partial \boldsymbol{W}} \neq 0
$$

Moreover, the characteristic of $E(\boldsymbol{W})$ is that it changes rapidly, which also implies the distance from the best solution $\boldsymbol{W}^{*}$. To increase the speed of iteration, by referring to the iteration value of the previous weight value matrix $\Delta \boldsymbol{W}(n-1)$, we can increase the distance of the weight value matrix displacement. Therefore, the $\Delta \boldsymbol{W}(n)$ of the modified equation is

$$
\Delta \boldsymbol{W}(n)=[\alpha+\beta \times \Delta \boldsymbol{W}(n-1)+\eta \times e(n)] \times \boldsymbol{X}(n) .
$$

In the equation, $\alpha, \beta$, and $\eta$ are all unknown scalar coefficients, and $\eta$ value can be chosen from the range given by Eq. (27). After that, according to the following equation, after the simulation analysis, the $\alpha$ and $\beta$ values are selected to microrepair the overlaying direction (Fig. 15).

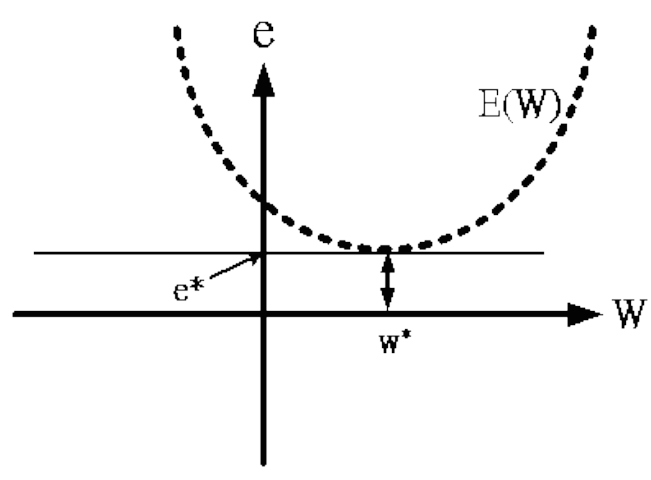

Fig. 14. Distribution of object function $E(\boldsymbol{W})$.

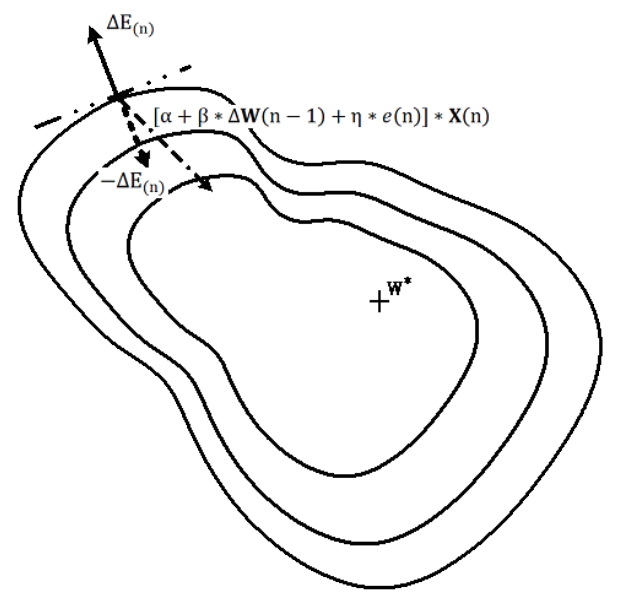

Fig. 15. Analysis of the learning rate of ADALINE artificial neural network. 


$$
\frac{y(n+1)-y(n)}{\left\lceil\frac{\|\boldsymbol{X}\|}{2}\right\rceil+1}<-[\alpha+\beta \times \Delta \boldsymbol{W}(n-1)+\eta \times e(n)]
$$

The previous processes have been explored for the tracking of analog signals, which have little effect on the transmission of digital signals. As long as the appropriate threshold $\varepsilon$ is set, it can be applied to the transmission of digital signals. Therefore, at the end of the inference, a conversion function $f(t)$ will be defined to convert analog signals to digital signals.

$$
f(t)= \begin{cases}1, & \text { if } \boldsymbol{W} \leq \varepsilon \\ 0, & \text { if } \boldsymbol{W}>\varepsilon\end{cases}
$$

\section{Simulation and Implementation}

To verify the correctness and feasibility of this research theory, as shown in Fig. 16, we constructed the smart motor drive platform hardware according to Fig. 12 and smart motors A-D, which respectively represent the smart motors 1 to 4 (Smart Motor $1-n$ ), as shown in Fig. 12. The labels A and B shown in Fig. 16 are the smart motors packaged with three-phase induction motors, respectively simulating no load (B, also known as ACM2) and $0.1 \mathrm{Nm}$ load (A, also known as ACM1). On the other hand, the two smart motors packaged with DC motors (C and D, as shown in Fig. 16) respectively simulate no load (C, also known as DCM2) and $0.1 \mathrm{Nm}$ load (D, also known as DCM1). The front-side controller is responsible for outputting PKM power waves and receiving the sent back data. Moreover, the front-side controller also has a Bluetooth communication interface, which can receive commands sent by a mobile phone, and can also transmit data to the mobile phone. Besides, waveform data such as PKM power waves are transmitted to a personal computer via the front-side controller at the same time. The record obtained via MATLAB/Simulink ${ }^{\circledR}$ and the current signals taken from $T_{r}$ will transmit the results to the front controller via a USB interface by the tracking and analysis of the ADALINE

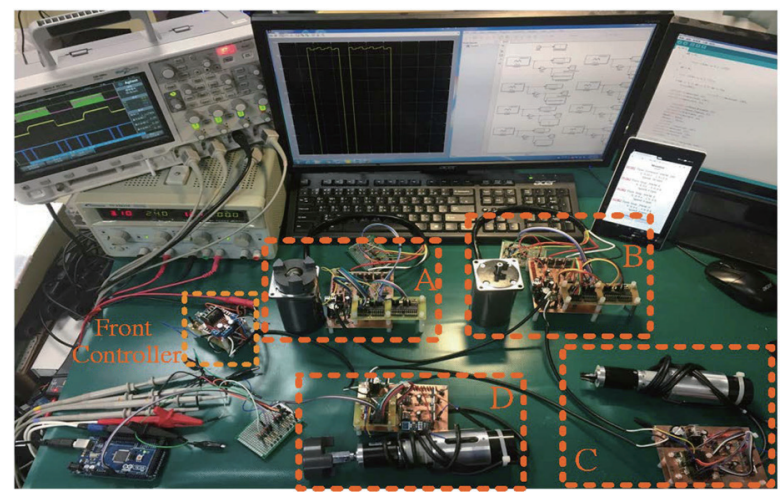

Fig. 16. (Color online) Smart motor drive platform hardware. 
neural network. Then, it outputs the controlled PKM power wave at $T_{t}$ in the next period. The digital waver displays an instant electrical signal.

Let $\varepsilon, \alpha, \beta$, and $\eta$ be $0.01,0.0001023,0.0015$, and 0.0618 , respectively. As shown in Figs. 17-22, we try to prove the ADALINE artificial neural network proposed in this study, and track the channel signals simulated by the weight matrix $\boldsymbol{W}$. Figures 17 and 18 are used to simulate different analog signals. Thereby, we can obtain the tracking results of amplitude-sized signals. Figures 19 and 20 are used to simulate the tracking results for different digital signals. By observing the tracking data, we realized that the ADALINE artificial neural network converges nearly after overlaying three times. As shown in Figs. 21 and 22, by using the ADALINE artificial neural network, we can simulate the single-channel and three-phase communication systems, respectively, to transmit dynamic waveforms. Figure 23 shows the waveforms that smart motor $\mathrm{C}$ was driven depending on the command from the front controller and the sentback information from smart motor $\mathrm{C}$ in the current waveform received by the front controller.

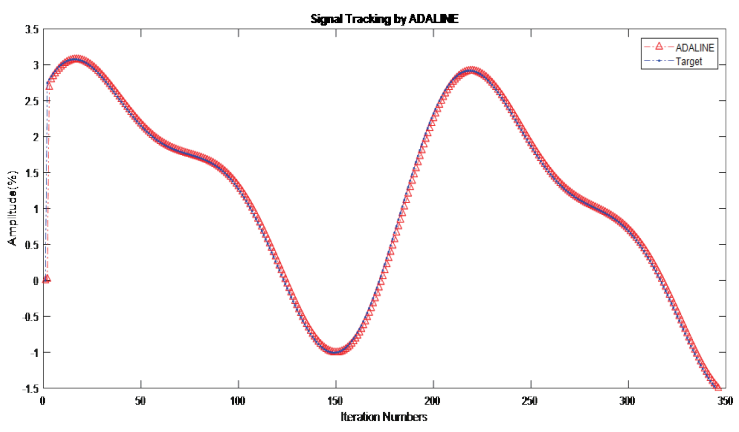

(a)

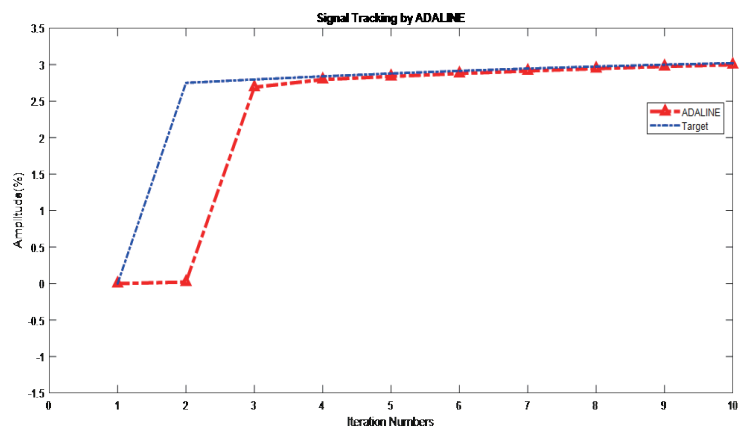

(b)

Fig. 17. (Color online) $\boldsymbol{W}=[1,0.4,0,1.5,1,0,0,0,0.7]$ simulating tracking results of eight smart motors transmitting ADALINE data. (a) Tracking waveform. (b) Overlay convergence result.

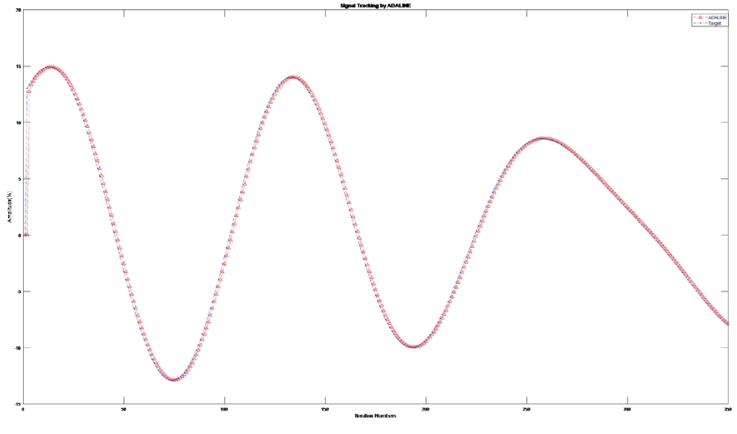

(a)

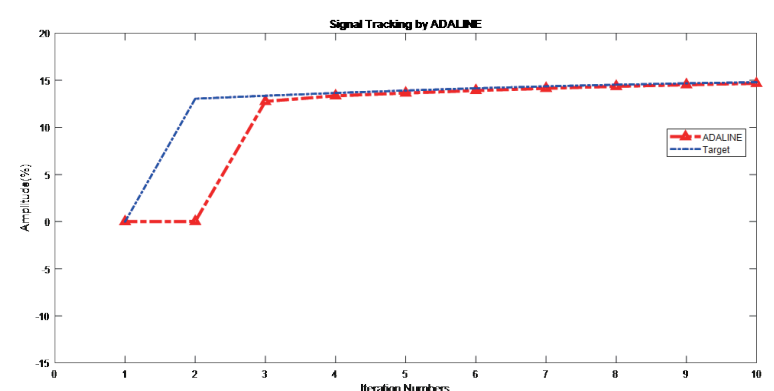

(b)

Fig. 18. (Color online) $\boldsymbol{W}=[1,0.4,0,1.5,1,0,10,5,0.7]$ simulating tracking results of eight smart motors transmitting ADALINE data. (a) Tracking waveform. (b) Overlay convergence result. 


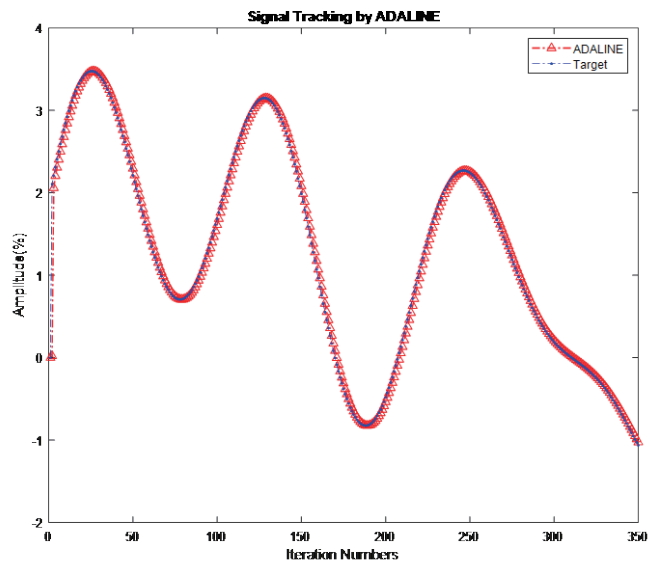

(a)

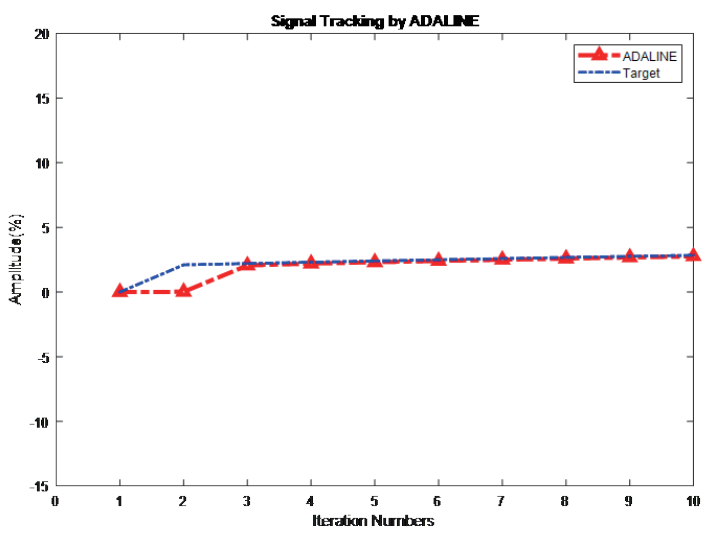

(b)

Fig. 19. (Color online) $\boldsymbol{W}=[1,1,0,1,0,0,1,1,0]$ simulating tracking results of eight smart motors transmitting ADALINE data. (a) Tracking waveform. (b) Overlay convergence result.

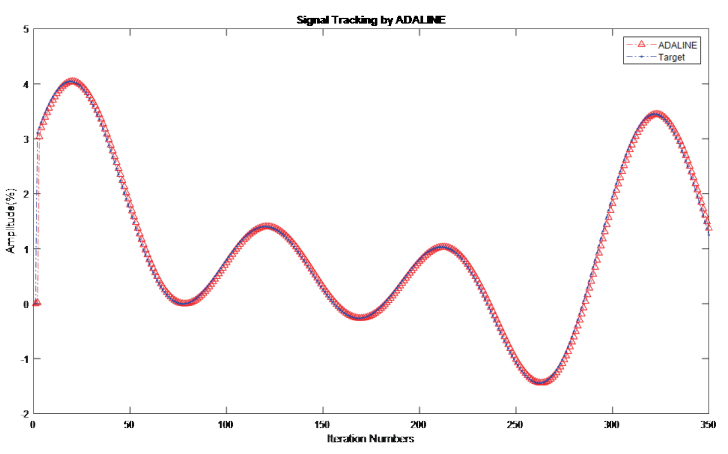

(a)

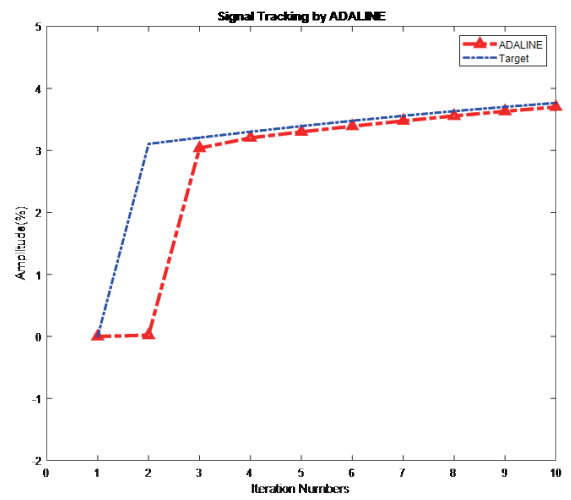

(b)

Fig. 20. (Color online) $\boldsymbol{W}=[1,0,1,0,0,1,0,1,1]$ simulating tracking results of eight smart motors transmitting ADALINE data. (a) Tracking waveform. (b) Overlay convergence result.

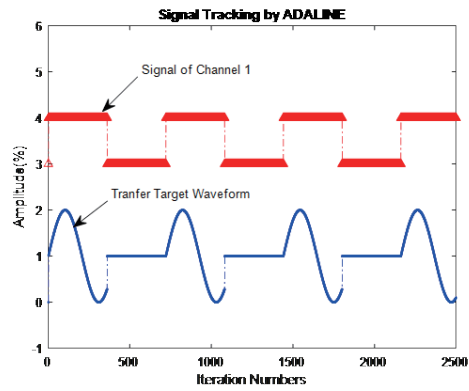

(a)

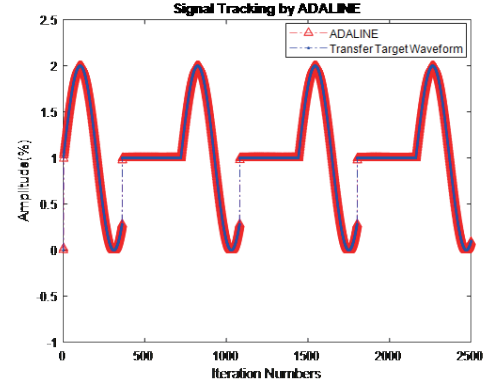

(b)

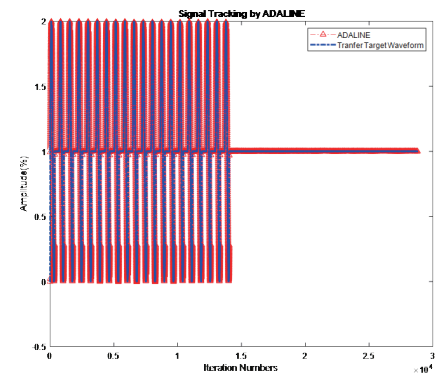

(c)

Fig. 21. (Color online) Simulation of communication using one channel for ADALINE signal from first channels. Transmission and tracking results: (a) waveform before and after conversion, (b) result of overlay convergence, and (c) transfer waveform. 


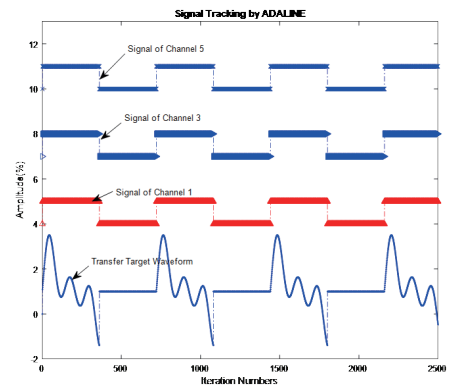

(a)

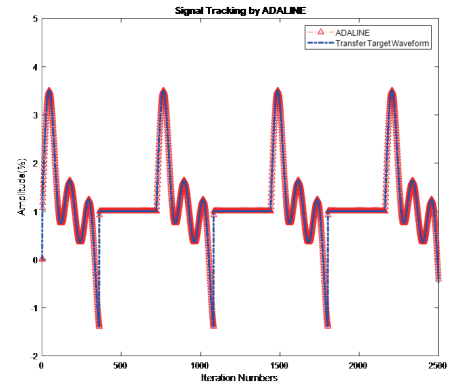

(b)

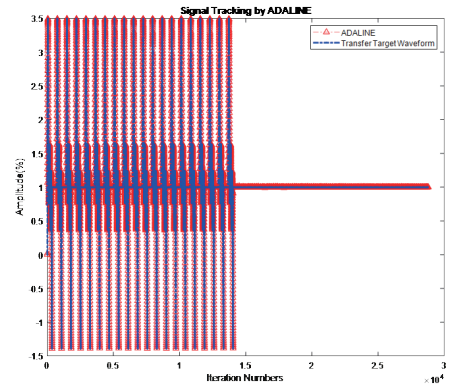

(c)

Fig. 22. (Color online) Simulation of three-channel communication for the ADALINE signal from the 1st, 3rd, and 5th channels. Transmitting and tracking results: (a) waveform before and after conversion, (b) results of overlaying convergence, and (c) transfer waveform.

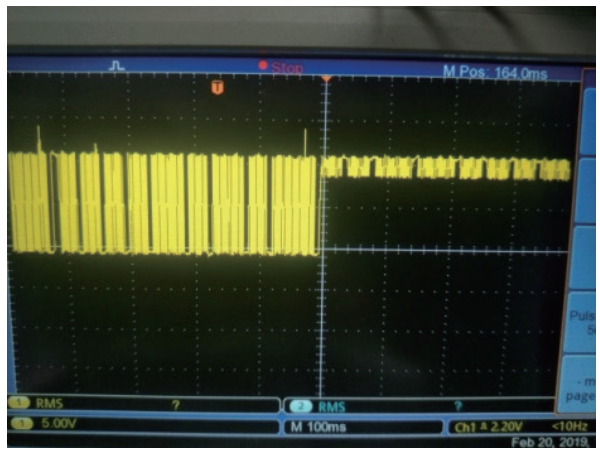

(a)

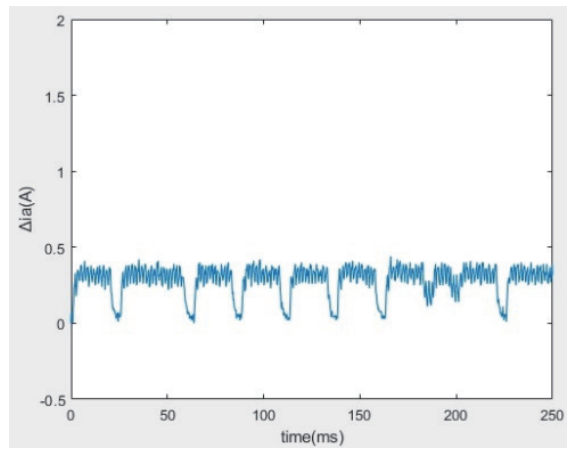

(b)

Fig. 23. (Color online) Front controller transfer command "00001010" and smart motor C carrier current waveform: (a) voltage waveforms during both $T_{t}$ and $T_{r}$ and (b) current returning waveform received by the front controller using current sensor.

Figure 24 shows the smart motor $\mathrm{D}$ waveform during the $T_{t}$ and $T_{r}$ procedures; in this case, the whole system seems to be changed into a digital system, so that the sent-back current signal can be encoded by a PK coding strategy. Figure 25 shows both the data sent during $T_{t}$ and the data fed back during $T_{r}$. In other words, the binary data "00001010" was changed to the PWM power wave during $T_{t}$, and then the sent-back data current waveform was detected at the DC busbar, and it will be identified by the ADALINE artificial neural networks using Matlab package software at the front controller.

By observing the simulation and implementation of data waveforms, we concluded that the methods and research direction of this study are feasible, and the response time is followed by the frequency of the $\omega$ sampling of the input variable $\boldsymbol{X}$ in the ADALINE artificial neural network, which can be completed at about three sampling points. Such an architecture allows multiple smart motors to act on the drive platform for faster operation. 


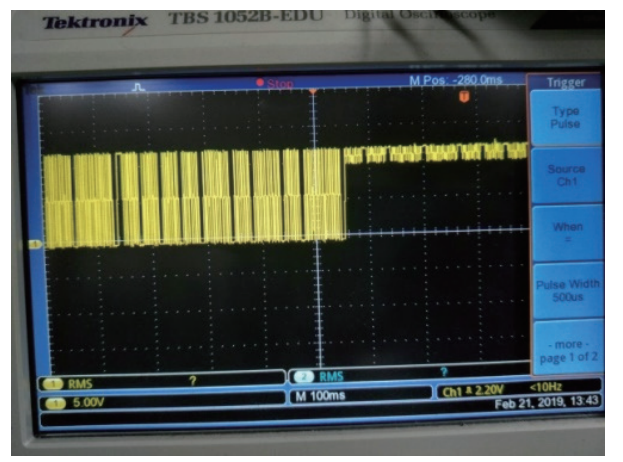

(a)

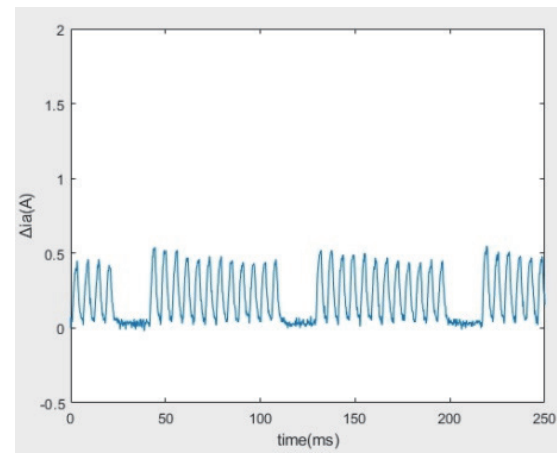

(b)

Fig. 24. (Color online) Front controller transfer command "00001010" and smart motor D carrier current waveform: (a) voltage waveforms during both $T_{t}$ and $T_{r}$ and (b) current returning waveform received by front controller using current sensor.

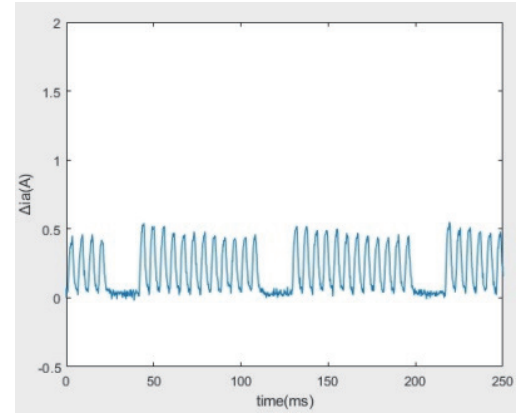

(a)

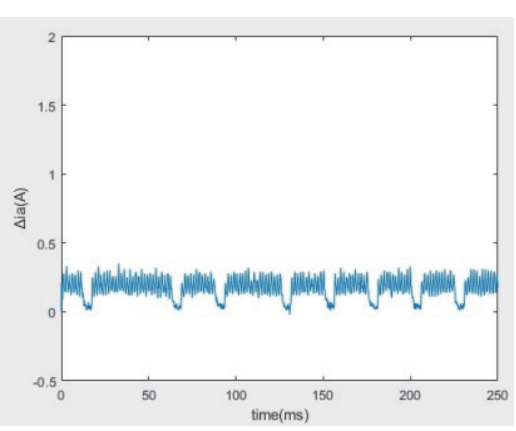

(b)

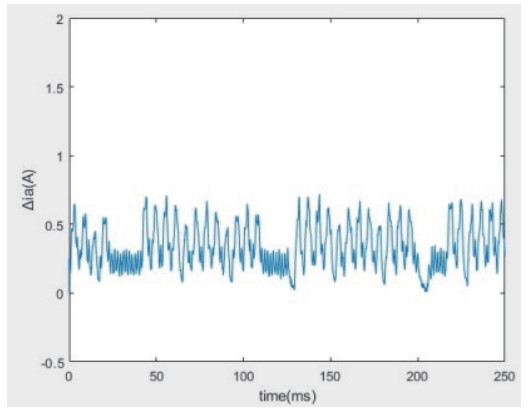

(c)

Fig. 25. (Color online) Current waveform sent back "01000011" to the front controller from both smart motor A and smart motor B by PKM strategy. (a) $x_{3}=\sin (2 \pi \times 200) t$, (b) $x_{15}=\sin (2 \pi \times 700) t$, and (c) mixed waveform.

\section{Conclusions}

In this study, we proposed a new smart motor drive architecture, which includes heterogeneous motors in smart motors and eliminates the need for additional communication modules. PKM power waves can provide power and communication. In addition to reducing interference and random changes in the number of motor drives, the final objective is to virtualize the motor drive and simplify the complexity of the multimotor system cooperative drive. Besides, in this study, we use the PKM power wave, which is simple in coding, faulttolerant, and has communication capability. It replaces the traditional PWM electric wave drive motor technology and is used as the communication interface of the smart motor drive platform. It also combines the ADALINE artificial neural network and sensors by establishing a multichannel communication platform that can send back information of multiple smart motors at the same time, directly increasing the speed of communication and indirectly increasing the overall response time of multiple motors, which is another significant contribution. The 
smart motor drive platform can be compatible with existing motor drive systems and is capable of integrating traditional devices without communication functions into the network, which is another contribution. In the future, the smart motor drive platform can be combined with AI technology and automatic adjustment parameters to power the learning platform, which is applied to the smart solar tracking platform and can provide power and communication resources to a drone. It can also be used to automatically repair drones and increase their endurance.

\section{Acknowledgments}

This work was supported by the Ministry of Science and Technology, Taiwan, under contract number MOST 108-2221-E-167-010, duration: August 1, 2019-July 31, 2020.

\section{References}

1 T. Tsuji, A. Jazidie, and M. Kaneko: IEEE Syst. Man. Cybern. Part B Cybern. 27 (1997) 862. https://doi. $\operatorname{org} / 10.1109 / 3477.623238$

2 B. Huang, Z. Li, X. Wu, A. Ajoudani, A. Bicchi, and J. Liu: IEEE Sys. Man. Cybern. Syst. 49 (2019) 954. https://doi.org/10.1109/TSMC.2017.2706694

3 C. Zhang, M. Niu, J. He, K. Zhao, H. Wu, and M. Zhang: IEEE Access 5 (2017) 197. https://doi.org/10.1109/ ACCESS.2016.2636224

4 H. Su, X. Wang, and Z. Lin: Autom. Contr. 54 (2009) 293. https://doi.org/10.1109/TAC.2008.2010897

5 B. Fidan, V. Gazi, S. Zhai, N. Cen, and E. Karata: Ind. Elec. 60 (2013) 5781. https://doi.org/10.1109/ TIE.2012.2236996.

6 M. Mengoni, A. Tani, L. Zarri, G. Serra, and D. Casadei: Ind. App. 48 (2012) 2048. https://doi.org/10.1109/ TIA.2012.2226420

7 T. Shi, H. Liu, Q. Geng, and C. Xia: Elec. Power App. 10 (2016) 451. https://doi.org/10.1049/iet-epa.2015.0515

8 T. Boller, J. Holtz, and A. K. Rathore: Ind. App. 48 (2012) 1610. https://doi.org/10.1109/ECCE.2011.6064229

9 G. Poddar and M. K. Sahu: Power Elec. 24 (2009) 1182. https://doi.org/10.1109/TPEL.2009.2013860

10 M. A. Mannah, N. Ginot, and C. Batard: Ind. Elec. 61 (2014) 4238. https://doi.org/10.1109/TIE. 2013.2288189

11 A. Mehrizi-Sani and S. Filizadeh: Ind. Elec. 56 (2009) 2894. https://doi.org/10.1109/TIE.2008.2008997

12 A. Tomar, Y. R. Sood, and D. S. Tomar: Ind. Info. 11 (2015) 132. https://doi.org/10.1109/TII.2014.2378734

13 S. L. Stevan, L. d. R. Farias, M. F. C. Barreto, and M. O. Leme: IEEE Latin America Trans. 14 (2016) 4241. https://doi.org/10.1109/TLA. 2016.7786300

14 V. Dégardin, K. Kilani, L. Koné, M. Liénard, and P. Degauque: Ind. Elec. 61 (2014) 4816. https://doi. org/10.1109/TIE.2013.2293697

15 W. C. Pu, H. C. Chiung, and J. J. Yang: proc. CACS 2016 (2016) 88.

16 W. C. Pu, M. L. Hung, and H. C. Chiung: Proc. ICASI (2015) 461.

17 W. C. Pu: Elimination of Power System Harmonics Using Harmonic Tracer in Active Filter (Feng Chia University, 1999) p. 10.

18 Z. Jin-xing, P. Wen-Cheng, and C. Qimin: Proc. 19th Symp. Power Engineering (1998) 803

19 M. Qasim, P. Kanjiya, and V. Khadkikar: Power Elec. 29 (2014) 6383. https://doi.org/10.1109/ TPEL.2014.2302539

20 G. Zhang, G. Wang, D. Xu, and Nannan Zhao: Power Elec. 31 (2016) 1450. https://doi.org/10.1109/ TPEL.2015.2424256 


\begin{abstract}
About the Authors
Wen-Cheng Pu received his Ph.D. degree from National Taiwan University of Science and Technology, Taipei, Taiwan, ROC, in 2010. He is currently an associate professor at the Department of Electrical Engineering, National Chin Yi University of Technology, Taichung, Taiwan, ROC. His current research interests include speed estimation of motor drives, automatic technology, and design of microcontroller-based motor drive.

Yu-Dian Lin joined the master's program in electrical engineering of National Chin-Yi University of Technology, Taiwan, in July 2018. His main research focus is on automatic technology and drive technology.

Jian-Xing Wu received his Ph.D. degree from the Department of Biomedical Engineering at National Cheng Kung University in 2014. He has been an assistant professor of the Department of Electrical Engineering, National Chin-Yi University of Technology, Taichung City, Taiwan, since 2019.
\end{abstract}
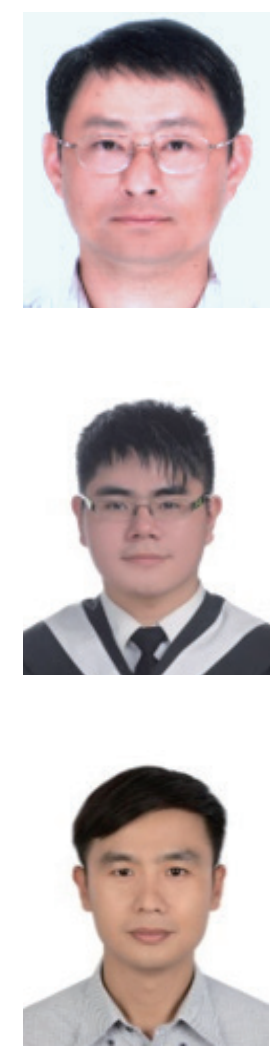

\title{
Appendix
}

Motor parameters

\begin{tabular}{lcccllc}
\hline & Voltage $(\mathrm{V})$ & Speed $(\mathrm{r} / \mathrm{min})$ & $R_{a}(\Omega)$ & $L_{a}(\mathrm{H})$ & $J\left(\mathrm{~kg} \cdot \mathrm{m}^{2}\right)$ & $B\left(\mathrm{~N} \cdot \mathrm{s} / \mathrm{m}^{2}\right)$ \\
\hline DCM1 & 12 & 50 & 136.7 & 0.53 & 0.000454 & 0.000338 \\
DCM2 & 12 & 50 & 136.7 & 0.53 & 0.000454 & 0.000338 \\
ACM1 & 24 & 2400 & 25.3 & 0.308 & 0.000801 & 0.000924 \\
ACM2 & 24 & 2400 & 25.3 & 0.308 & 0.000801 & 0.000924 \\
\hline
\end{tabular}

